\title{
Abstract generalized fractional Landau inequalities over $\mathbb{R}$
}

\author{
GEORGE A. AnASTASSIOU*
}

ABSTRACT. We present uniform and $L_{p}$ mixed Caputo-Bochner abstract generalized fractional Landau inequalities over $\mathbb{R}$ of fractional orders $2<\alpha \leq 3$. These estimate the size of first and second derivatives of a composition with a Banach space valued function over $\mathbb{R}$. We give applications when $\alpha=2.5$.

Keywords: Abstract generalized fractional Landau inequality, right and left Caputo abstract generalized fractional derivatives.

2020 Mathematics Subject Classification: 26A33, 26D10, 26D15.

Dedicated to Professor Francesco Altomare, on occasion of his 70th birthday, with esteem and friendship.

\section{INTRODUCTION}

Let $p \in[1, \infty], I=\mathbb{R}_{+}$or $I=\mathbb{R}$ and $f: I \rightarrow \mathbb{R}$ is twice differentiable with $f, f^{\prime \prime} \in L_{p}(I)$, then $f^{\prime} \in L_{p}(I)$. Moreover, there exists a constant $C_{p}(I)>0$ independent of $f$, such that

$$
\left\|f^{\prime}\right\|_{p, I} \leq C_{p}(I)\|f\|_{p, I}^{\frac{1}{2}}\left\|f^{\prime \prime}\right\|_{p, I}^{\frac{1}{2}},
$$

where $\|\cdot\|_{p, I}$ is the $p$-norm on the interval $I$, see [1], [5]. The research on these inequalities started by E. Landau [10] in 1913. For the case of $p=\infty$, he proved that

$$
C_{\infty}\left(\mathbb{R}_{+}\right)=2 \text { and } C_{\infty}(\mathbb{R})=\sqrt{2}
$$

are the best constants in (1). In 1932, G. H. Hardy and J. E. Littlewood [7] proved (1) for $p=2$, with the best constants

$$
C_{2}\left(\mathbb{R}_{+}\right)=\sqrt{2} \text { and } C_{2}(\mathbb{R})=1 .
$$

In 1935, G. H. Hardy, E. Landau and J. E. Littlewood [8] showed that the best constants $C_{p}\left(\mathbb{R}_{+}\right)$ in (1) satisfies the estimate

$$
C_{p}\left(\mathbb{R}_{+}\right) \leq 2, \text { for } p \in[1, \infty),
$$

which yields $C_{p}(\mathbb{R}) \leq 2$ for $p \in[1, \infty)$.

In fact, in [6] and [9] was shown that $C_{p}(\mathbb{R}) \leq \sqrt{2}$. We need the following concepts from abstract generalized fractional calculus. Our integrals next are of Bochner type [11]. We need the following definition. 
Definition 1.1. ([4], p. 104) Let $[a, b] \subset \mathbb{R},(X,\|\cdot\|)$ a Banach space, $g \in C^{1}([a, b])$ and increasing, $f \in C([a, b], X), \nu>0$. We define the left Riemann-Liouville generalized fractional Bochner integral operator

$$
\left(J_{a ; g}^{\nu} f\right)(x):=\frac{1}{\Gamma(\nu)} \int_{a}^{x}(g(x)-g(z))^{\nu-1} g^{\prime}(z) f(z) d z,
$$

$\forall x \in[a, b]$, where $\Gamma$ is the gamma function. The last integral is of Bochner type. Since $f \in C([a, b], X)$, then $f \in L_{\infty}([a, b], X)$. By Theorem 4.10, p. 98, [4], we get that $\left(J_{a ; g}^{\nu} f\right) \in C([a, b], X)$. Above we set $J_{a ; g}^{0} f:=f$ and see that $\left(J_{a ; g}^{\nu} f\right)(a)=0$.

We need the following definition.

Definition 1.2. ([4], p. 105) Let $[a, b] \subset \mathbb{R},(X,\|\cdot\|)$ a Banach space, $g \in C^{1}([a, b])$ and increasing, $f \in C([a, b], X), \nu>0$. We define the right Riemann-Liouville generalized fractional Bochner integral operator

$$
\left(J_{b-; g}^{\nu} f\right)(x):=\frac{1}{\Gamma(\nu)} \int_{x}^{b}(g(z)-g(x))^{\nu-1} g^{\prime}(z) f(z) d z,
$$

$\forall x \in[a, b]$, where $\Gamma$ is the gamma function. The last integral is of Bochner type. Since $f \in C([a, b], X)$, then $f \in L_{\infty}([a, b], X)$. By Theorem 4.11, p. 101, [4], we get that $\left(J_{b-; g}^{\nu} f\right) \in C([a, b], X)$. Above we set $J_{b-; g}^{0} f:=f$ and see that $\left(J_{b-; g}^{\nu} f\right)(b)=0$.

We also need the following definition.

Definition 1.3. ([4], $p$. 106) Let $\alpha>0,\lceil\alpha\rceil=n,\lceil\cdot\rceil$ the ceiling of the number. Let $f \in C^{n}([a, b], X)$, where $[a, b] \subset \mathbb{R}$, and $(X,\|\cdot\|)$ is a Banach space. Let $g \in C^{1}([a, b])$, strictly increasing, such that $g^{-1} \in C^{n}([g(a), g(b)])$. We define the left generalized $g$-fractional derivative $X$-valued of $f$ of order $\alpha$ as follows:

$$
\left(D_{a+; g}^{\alpha} f\right)(x):=\frac{1}{\Gamma(n-\alpha)} \int_{a}^{x}(g(x)-g(t))^{n-\alpha-1} g^{\prime}(t)\left(f \circ g^{-1}\right)^{(n)}(g(t)) d t,
$$

$\forall x \in[a, b]$. The last integral is of Bochner type. Ordinary vector valued derivative is as in [12], similar to numerical one. If $\alpha \notin \mathbb{N}$, by Theorem 4.10, p. 98, [4], we have that $\left(D_{a+; g}^{\alpha} f\right) \in C([a, b], X)$. We see that

$$
\left(J_{a ; g}^{n-\alpha}\left(\left(f \circ g^{-1}\right)^{(n)} \circ g\right)\right)(x)=\left(D_{a+; g}^{\alpha} f\right)(x), \quad \forall x \in[a, b] .
$$

We set

$$
\begin{aligned}
D_{a+; g}^{n} f(x): & \left(\left(f \circ g^{-1}\right)^{n} \circ g\right)(x) \in C([a, b], X), n \in \mathbb{N}, \\
& D_{a+; g}^{0} f(x)=f(x), \forall x \in[a, b] .
\end{aligned}
$$

When $g=i d$, then

$$
D_{a+; g}^{\alpha} f=D_{a+; i d}^{\alpha} f=D_{* a}^{\alpha} f,
$$

the usual left $X$-valued Caputo fractional derivative, see [4, Chapter 1].

We mention the following definition. 
Definition 1.4. ([4], $p .107)$ Let $\alpha>0,\lceil\alpha\rceil=n,\lceil\cdot\rceil$ the ceiling of the number. Let $f \in C^{n}([a, b], X)$, where $[a, b] \subset \mathbb{R}$, and $(X,\|\cdot\|)$ is a Banach space. Let $g \in C^{1}([a, b])$, strictly increasing, such that $g^{-1} \in C^{n}([g(a), g(b)])$. We define the right generalized $g$-fractional derivative $X$-valued of $f$ of order $\alpha$ as follows:

$$
\left(D_{b-; g}^{\alpha} f\right)(x):=\frac{(-1)^{n}}{\Gamma(n-\alpha)} \int_{x}^{b}(g(t)-g(x))^{n-\alpha-1} g^{\prime}(t)\left(f \circ g^{-1}\right)^{(n)}(g(t)) d t
$$

$\forall x \in[a, b]$. The last integral is of Bochner type. If $\alpha \notin \mathbb{N}$, by Theorem 4.11, $p .101,[4]$, we have that $\left(D_{b-; g}^{\alpha} f\right) \in C([a, b], X)$. We see that

$$
J_{b-; g}^{n-\alpha}\left((-1)^{n}\left(f \circ g^{-1}\right)^{(n)} \circ g\right)(x)=\left(D_{b-; g}^{\alpha} f\right)(x), a \leq x \leq b .
$$

We set

$$
\begin{gathered}
D_{b-; g}^{n} f(x):=(-1)^{n}\left(\left(f \circ g^{-1}\right)^{n} \circ g\right)(x) \in C([a, b], X), n \in \mathbb{N}, \\
D_{b-; g}^{0} f(x):=f(x), \quad \forall x \in[a, b] .
\end{gathered}
$$

When $g=i d$, then

$$
D_{b-; g}^{\alpha} f(x)=D_{b-; i d}^{\alpha} f(x)=D_{b-}^{\alpha} f,
$$

the usual right $X$-valued Caputo fractional derivative, see [4, Chapter 2].

We mention the generalized left fractional Taylor formula:

Theorem 1.1. ([4], $p$. 107) Let $\alpha>0, n=\lceil\alpha\rceil$, and $f \in C^{n}([a, b], X)$, where $[a, b] \subset \mathbb{R}$ and $(X,\|\cdot\|)$ is a Banach space. Let $g \in C^{1}([a, b])$, strictly increasing, such that $g^{-1} \in C^{n}([g(a), g(b)])$, $a \leq x \leq b$. Then,

$$
\begin{aligned}
f(x) & =f(a)+\sum_{i=1}^{n-1} \frac{(g(x)-g(a))^{i}}{i !}\left(f \circ g^{-1}\right)^{(i)}(g(a)) \\
& +\frac{1}{\Gamma(\alpha)} \int_{a}^{x}(g(x)-g(t))^{\alpha-1} g^{\prime}(t)\left(D_{a+; g}^{\alpha} f\right)(t) d t \\
& =f(a)+\sum_{i=1}^{n-1} \frac{(g(x)-g(a))^{i}}{i !}\left(f \circ g^{-1}\right)^{(i)}(g(a)) \\
& +\frac{1}{\Gamma(\alpha)} \int_{g(a)}^{g(x)}(g(x)-z)^{\alpha-1}\left(\left(D_{a+; g}^{\alpha} f\right) \circ g^{-1}\right)(z) d z .
\end{aligned}
$$

We also mention the generalized right fractional Taylor formula:

Theorem 1.2. ([4], $p$. 108) Let $\alpha>0, n=\lceil\alpha\rceil$, and $f \in C^{n}([a, b], X)$, where $[a, b] \subset \mathbb{R}$ and $(X,\|\cdot\|)$ is a Banach space. Let $g \in C^{1}([a, b])$, strictly increasing, such that $g^{-1} \in C^{n}([g(a), g(b)])$, 
$a \leq x \leq b$. Then,

$$
\begin{aligned}
f(x) & =f(b)+\sum_{i=1}^{n-1} \frac{(g(x)-g(b))^{i}}{i !}\left(f \circ g^{-1}\right)^{(i)}(g(b)) \\
& +\frac{1}{\Gamma(\alpha)} \int_{x}^{b}(g(t)-g(x))^{\alpha-1} g^{\prime}(t)\left(D_{b-; g}^{\alpha} f\right)(t) d t \\
& =f(b)+\sum_{i=1}^{n-1} \frac{(g(x)-g(b))^{i}}{i !}\left(f \circ g^{-1}\right)^{(i)}(g(b)) \\
& +\frac{1}{\Gamma(\alpha)} \int_{g(x)}^{g(b)}(z-g(x))^{\alpha-1}\left(\left(D_{b-; g}^{\alpha} f\right) \circ g^{-1}\right)(z) d z .
\end{aligned}
$$

By convention, we suppose that

$$
\left(D_{x_{0}+; g}^{\alpha} f\right)(x)=0, \text { for } x<x_{0},
$$

$$
\left(D_{x_{0}-; g}^{\alpha} f\right)(x)=0, \text { for } x>x_{0},
$$

for any $x, x_{0} \in[a, b]$.

The author has already done an extensive amount of work on fractional Landau inequalities, see [3], and on abstract fractional Landau inequalities, see [4]. However, there the proving methods came out of applications of fractional Ostrowski inequalities ([2], [4]) and the derived inequalities were for small fractional orders, i.e. $\alpha \in(0,1)$. Usually there the domains where $[A,+\infty)$ or $(-\infty, B]$, with $A, B \in \mathbb{R}$ and in one mixed case the domain was all of $\mathbb{R}$.

In this work with less assumptions, we establish uniform and $L_{p}$ type mixed Caputo-Bochner abstract generalized fractional Landau inequalities over $\mathbb{R}$ for fractional orders $2<\alpha \leq 3$. The method of proving is based on left and right Caputo-Bochner generalized fractional Taylor's formulae with integral remainder, see Theorems 1.1,1.2. We give also applications for $\alpha=2.5$. Certainly, we are also inspired by [3], [4].

\section{MAIN RESULTS}

We give the following abstract mixed generalized fractional Landau inequalities over $\mathbb{R}$.

Theorem 2.3. Let $2<\alpha \leq 3$ and $f \in C^{3}(\mathbb{R}, X)$, where $(X,\|\cdot\|)$ is a Banach space. Let $g \in C^{1}(\mathbb{R})$, strictly increasing, such that $g^{-1} \in C^{3}(g(\mathbb{R}))$. We assume that \|\|$f\|\|_{\infty, \mathbb{R}}<\infty$ and that

$$
\begin{aligned}
K:= & \max \left\{\|\|\left(\left(D_{a+; g}^{\alpha} f\right) \circ g^{-1}\right)(z)\|\|_{\infty, \mathbb{R} \times g(\mathbb{R})},\right. \\
& \left.\|\|\left(\left(D_{a-; g}^{\alpha} f\right) \circ g^{-1}\right)(z)\|\|_{\infty, \mathbb{R} \times g(\mathbb{R})}\right\}<\infty,
\end{aligned}
$$

where $(a, z) \in \mathbb{R} \times g(\mathbb{R})$. Then,

$$
\|\|\left(f \circ g^{-1}\right)^{\prime} \circ g\|\|_{\infty, \mathbb{R}} \leq \alpha\left(\frac{K}{\Gamma(\alpha+1)}\right)^{\frac{1}{\alpha}}\left(\frac{\|\| f\|\|_{\infty, \mathbb{R}}}{\alpha-1}\right)^{\frac{\alpha-1}{\alpha}}
$$

and

$$
\|\|\left(f \circ g^{-1}\right)^{\prime \prime} \circ g\|\|_{\infty, \mathbb{R}} \leq \alpha\left(\frac{K}{\Gamma(\alpha+1)}\right)^{\frac{2}{\alpha}}\left(\frac{4\|\| f\|\|_{\infty, \mathbb{R}}}{\alpha-2}\right)^{\frac{\alpha-2}{\alpha}} .
$$

That is,

$$
\|\|\left(f \circ g^{-1}\right)^{\prime} \circ g\|\|_{\infty, \mathbb{R}},\|\|\left(f \circ g^{-1}\right)^{\prime \prime} \circ g\|\|_{\infty, \mathbb{R}}<\infty
$$


Proof. Here $2<\alpha \leq 3$, i.e. $\lceil\alpha\rceil=3$. Let $f \in C^{3}(\mathbb{R}, X)$, where $(X,\|\cdot\|)$ is a Banach space, $a \in \mathbb{R}$ is fixed momentarily. We need the following abstract generalized fractional Taylor formulae for $n=3$. By Theorem 1.1, we get

$$
\begin{aligned}
f(x)-f(a) & =(g(x)-g(a))\left(f \circ g^{-1}\right)^{\prime}(g(a))+\frac{(g(x)-g(a))^{2}}{2}\left(f \circ g^{-1}\right)^{\prime \prime}(g(a)) \\
& +\frac{1}{\Gamma(\alpha)} \int_{g(a)}^{g(x)}(g(x)-z)^{\alpha-1}\left(\left(D_{a+; g}^{\alpha} f\right) \circ g^{-1}\right)(z) d z, \quad \forall x \geq a .
\end{aligned}
$$

And by Theorem 1.2, we get

$$
\begin{aligned}
f(x)-f(a) & =(g(x)-g(a))\left(f \circ g^{-1}\right)^{\prime}(g(a))+\frac{(g(x)-g(a))^{2}}{2}\left(f \circ g^{-1}\right)^{\prime \prime}(g(a)) \\
& +\frac{1}{\Gamma(\alpha)} \int_{g(x)}^{g(a)}(z-g(x))^{\alpha-1}\left(\left(D_{a-; g}^{\alpha} f\right) \circ g^{-1}\right)(z) d z, \quad \forall x \leq a .
\end{aligned}
$$

Let $x_{1}>a$, then

$$
\begin{aligned}
& \left(g\left(x_{1}\right)-g(a)\right)\left(f \circ g^{-1}\right)^{\prime}(g(a))+\frac{\left(g\left(x_{1}\right)-g(a)\right)^{2}}{2}\left(f \circ g^{-1}\right)^{\prime \prime}(g(a)) \\
= & \left(f\left(x_{1}\right)-f(a)\right)-\frac{1}{\Gamma(\alpha)} \int_{g(a)}^{g\left(x_{1}\right)}\left(g\left(x_{1}\right)-z\right)^{\alpha-1}\left(\left(D_{a+; g}^{\alpha} f\right) \circ g^{-1}\right)(z) d z=: A,
\end{aligned}
$$

and let $x_{2}<a$, then

$$
\begin{aligned}
& \left(g\left(x_{2}\right)-g(a)\right)\left(f \circ g^{-1}\right)^{\prime}(g(a))+\frac{\left(g\left(x_{2}\right)-g(a)\right)^{2}}{2}\left(f \circ g^{-1}\right)^{\prime \prime}(g(a)) \\
= & \left(f\left(x_{2}\right)-f(a)\right)-\frac{1}{\Gamma(\alpha)} \int_{g\left(x_{2}\right)}^{g(a)}\left(z-g\left(x_{2}\right)\right)^{\alpha-1}\left(\left(D_{a-; g}^{\alpha} f\right) \circ g^{-1}\right)(z) d z=: B .
\end{aligned}
$$

Let $h>0$, we can choose $x_{1}$ such that $g\left(x_{1}\right)-g(a)=h$ and we can choose $x_{2}$ such that $g(a)-g\left(x_{2}\right)=h$. That is $g\left(x_{1}\right)=g(a)+h$ and $g\left(x_{2}\right)=g(a)-h$, and $g\left(x_{2}\right)-g(a)=-h$. Furthermore, it holds $g\left(x_{2}\right)-g\left(x_{1}\right)=-2 h$. We can rewrite (23) as

$$
h\left(f \circ g^{-1}\right)^{\prime}(g(a))+\frac{h^{2}}{2}\left(f \circ g^{-1}\right)^{\prime \prime}(g(a))=A,
$$

and we can rewrite (24) as

$$
-h\left(f \circ g^{-1}\right)^{\prime}(g(a))+\frac{h^{2}}{2}\left(f \circ g^{-1}\right)^{\prime \prime}(g(a))=B .
$$

Solving the system of (25) and (26), we find

$$
\left(f \circ g^{-1}\right)^{\prime}(g(a))=\frac{A-B}{2 h}
$$

and

We assumed that

$$
\left(f \circ g^{-1}\right)^{\prime \prime}(g(a))=\frac{A+B}{h^{2}} .
$$

$$
\|\|\left(\left(D_{a+; g}^{\alpha} f\right) \circ g^{-1}\right)(z)\|\|_{\infty, \mathbb{R} \times g(\mathbb{R})},\|\|\left(\left(D_{a-; g}^{\alpha} f\right) \circ g^{-1}\right)(z)\|\|_{\infty, \mathbb{R} \times g(\mathbb{R})}<\infty .
$$

We obtain,

$$
\left\|\left(f \circ g^{-1}\right)^{\prime}(g(a))\right\|=\frac{1}{2 h}\|A-B\|
$$


and

(28)

$$
\left\|\left(f \circ g^{-1}\right)^{\prime \prime}(g(a))\right\| \leq \frac{1}{h^{2}}(\|A\|+\|B\|) .
$$

\section{We get}

(29)

$$
\begin{aligned}
\|A\| & =\left\|\left(f\left(x_{1}\right)-f(a)\right)-\frac{1}{\Gamma(\alpha)} \int_{g(a)}^{g\left(x_{1}\right)}\left(g\left(x_{1}\right)-z\right)^{\alpha-1}\left(\left(D_{a+; g}^{\alpha} f\right) \circ g^{-1}\right)(z) d z\right\| \\
& \leq 2\|\| f\|\|_{\infty, \mathbb{R}}+\frac{1}{\Gamma(\alpha)} \int_{g(a)}^{g\left(x_{1}\right)}\left(g\left(x_{1}\right)-z\right)^{\alpha-1}\left\|\left(\left(D_{a+; g}^{\alpha} f\right) \circ g^{-1}\right)(z)\right\| d z \\
& \leq 2\|\| f\|\|_{\infty, \mathbb{R}}+\frac{K}{\Gamma(\alpha)}\left(\int_{g(a)}^{g\left(x_{1}\right)}\left(g\left(x_{1}\right)-z\right)^{\alpha-1} d z\right) \\
& =2\|\| f\|\|_{\infty, \mathbb{R}}+\frac{K}{\Gamma(\alpha+1)}\left(g\left(x_{1}\right)-g(a)\right)^{\alpha}=2\|\| f\|\|_{\infty, \mathbb{R}}+\frac{K}{\Gamma(\alpha+1)} h^{\alpha} .
\end{aligned}
$$

That is,

(30)

$$
\|A\| \leq 2\|\| f\|\|_{\infty, \mathbb{R}}+\frac{K}{\Gamma(\alpha+1)} h^{\alpha}, \quad h>0 .
$$

Similarly, it holds

$$
\begin{aligned}
\|B\| & =\left\|\left(f\left(x_{2}\right)-f(a)\right)-\frac{1}{\Gamma(\alpha)} \int_{g\left(x_{2}\right)}^{g(a)}\left(z-g\left(x_{2}\right)\right)^{\alpha-1}\left(\left(D_{a-; g}^{\alpha} f\right) \circ g^{-1}\right)(z) d z\right\| \\
& \leq 2\|\| f\|\|_{\infty, \mathbb{R}}+\frac{1}{\Gamma(\alpha)} \int_{g\left(x_{2}\right)}^{g(a)}\left(z-g\left(x_{2}\right)\right)^{\alpha-1}\left\|\left(\left(D_{a-; g}^{\alpha} f\right) \circ g^{-1}\right)(z)\right\| d z \\
& \leq 2\|\| f\|\|_{\infty, \mathbb{R}}+\frac{K}{\Gamma(\alpha)}\left(\int_{g\left(x_{2}\right)}^{g(a)}\left(z-g\left(x_{2}\right)\right)^{\alpha-1} d z\right) \\
& =2\|\| f\|\|_{\infty, \mathbb{R}}+\frac{K}{\Gamma(\alpha+1)}\left(g(a)-g\left(x_{2}\right)\right)^{\alpha}=2\|\| f\|\|_{\infty, \mathbb{R}}+\frac{K}{\Gamma(\alpha+1)} h^{\alpha} .
\end{aligned}
$$

That is,

$$
\|B\| \leq 2\|\| f\|\|_{\infty, \mathbb{R}}+\frac{K}{\Gamma(\alpha+1)} h^{\alpha}, \quad h>0 .
$$

Furthermore, we have

$$
\|A\|+\|B\| \leq 4\|\| f\|\|_{\infty, \mathbb{R}}+\frac{2 K}{\Gamma(\alpha+1)} h^{\alpha}, \quad h>0 .
$$


We also notice that

(34)

$$
\begin{aligned}
\|A-B\| & =\| f\left(x_{1}\right)-f(a)-\frac{1}{\Gamma(\alpha)} \int_{g(a)}^{g\left(x_{1}\right)}\left(g\left(x_{1}\right)-z\right)^{\alpha-1}\left(\left(D_{a+; g}^{\alpha} f\right) \circ g^{-1}\right)(z) d z \\
& -f\left(x_{2}\right)+f(a)+\frac{1}{\Gamma(\alpha)} \int_{g\left(x_{2}\right)}^{g(a)}\left(z-g\left(x_{2}\right)\right)^{\alpha-1}\left(\left(D_{a-; g}^{\alpha} f\right) \circ g^{-1}\right)(z) d z \| \\
& \leq\left\|f\left(x_{1}\right)-f\left(x_{2}\right)\right\|+\frac{1}{\Gamma(\alpha)}\left[\int_{g(a)}^{g\left(x_{1}\right)}\left(g\left(x_{1}\right)-z\right)^{\alpha-1}\left\|\left(\left(D_{a+; g}^{\alpha} f\right) \circ g^{-1}\right)(z)\right\| d z\right. \\
& \left.+\int_{g\left(x_{2}\right)}^{g(a)}\left(z-g\left(x_{2}\right)\right)^{\alpha-1}\left\|\left(\left(D_{a-; g}^{\alpha} f\right) \circ g^{-1}\right)(z)\right\| d z\right] \\
& \leq 2\|\| f\|\|_{\infty, \mathbb{R}}+\frac{K}{\Gamma(\alpha)}\left[\int_{g(a)}^{g\left(x_{1}\right)}\left(g\left(x_{1}\right)-z\right)^{\alpha-1} d z+\int_{g\left(x_{2}\right)}^{g(a)}\left(z-g\left(x_{2}\right)\right)^{\alpha-1} d z\right] \\
& =2\|\| f\|\|_{\infty, \mathbb{R}}+\frac{K}{\Gamma(\alpha+1)}\left[\left(g\left(x_{1}\right)-g(a)\right)^{\alpha}+\left(g(a)-g\left(x_{2}\right)\right)^{\alpha}\right] \\
& =2\|\| f\|\|_{\infty, \mathbb{R}}+\frac{2 K h^{\alpha}}{\Gamma(\alpha+1)} .
\end{aligned}
$$

That is,

$$
\frac{\|A-B\|}{2} \leq\|\| f\|\|_{\infty, \mathbb{R}}+\frac{K h^{\alpha}}{\Gamma(\alpha+1)}, \quad h>0 .
$$

Consequently, we obtain

$$
\left\|\left(f \circ g^{-1}\right)^{\prime}(g(a))\right\| \stackrel{((28),(35))}{\leq} \frac{\|\| f\|\|_{\infty, \mathbb{R}}}{h}+\frac{K h^{\alpha-1}}{\Gamma(\alpha+1)}
$$

and

$$
\left\|\left(f \circ g^{-1}\right)^{\prime \prime}(g(a))\right\| \stackrel{((28),(33))}{\leq} \frac{4\|\| f\|\|_{\infty, \mathbb{R}}}{h^{2}}+\frac{2 K h^{\alpha-2}}{\Gamma(\alpha+1)},
$$

$h>0$, for any $a \in \mathbb{R}$. Hence,

$$
\|\|\left(f \circ g^{-1}\right)^{\prime} \circ g\|\|_{\infty, \mathbb{R}} \leq \frac{\|\| f\|\|_{\infty, \mathbb{R}}}{h}+\frac{K h^{\alpha-1}}{\Gamma(\alpha+1)}
$$

and

$$
\|\|\left(f \circ g^{-1}\right)^{\prime \prime} \circ g\|\|_{\infty, \mathbb{R}} \leq \frac{4\|\| f\|\|_{\infty, \mathbb{R}}}{h^{2}}+\frac{2 K h^{\alpha-2}}{\Gamma(\alpha+1)},
$$

true $\forall h>0,2<\alpha \leq 3$. Call

$$
\mu:=\|\| f\|\|_{\infty, \mathbb{R}}, \quad \theta=\frac{K}{\Gamma(\alpha+1)},
$$

both are greater than zero. Set also $\rho:=\alpha-1>1$. We consider the function

$$
y(h):=\frac{\mu}{h}+\theta h^{\rho}, \quad \forall h>0 .
$$

We have

$$
y^{\prime}(h)=-\frac{\mu}{h^{2}}+\rho \theta h^{\rho-1}=0,
$$


then

$$
\rho \theta h^{\rho+1}=\mu,
$$

with a unique solution

$$
h_{0}:=h_{\text {crit.no }}=\left(\frac{\mu}{\rho \theta}\right)^{\frac{1}{\rho+1}} .
$$

We have that

$$
y^{\prime \prime}(h)=2 \mu h^{-3}+\rho(\rho-1) \theta h^{\rho-2} .
$$

We observe that

$$
\begin{aligned}
y^{\prime \prime}\left(h_{0}\right) & =2 \mu\left(\frac{\mu}{\rho \theta}\right)^{-\frac{3}{\rho+1}}+\rho(\rho-1) \theta\left(\frac{\mu}{\rho \theta}\right)^{\frac{((\rho+1)-3)}{\rho+1}} \\
& =\left(\frac{\mu}{\rho \theta}\right)^{-\frac{3}{\rho+1}}[2 \mu+\mu(\rho-1)]=\mu\left(\frac{\mu}{\rho \theta}\right)^{-\frac{3}{\rho+1}}(\rho+1)>0 .
\end{aligned}
$$

Therefore, $y$ has a global minimum at $h_{0}=\left(\frac{\mu}{\rho \theta}\right)^{\frac{1}{\rho+1}}$, which is

$$
\begin{aligned}
y\left(h_{0}\right) & =\frac{\mu}{\left(\frac{\mu}{\rho \theta}\right)^{\frac{1}{\rho+1}}}+\theta\left(\frac{\mu}{\rho \theta}\right)^{\frac{\rho}{\rho+1}} \\
& =(\rho \theta)^{\frac{1}{\rho+1}} \frac{\mu}{\mu^{\frac{1}{\rho+1}}}+\frac{\theta \mu^{\frac{\rho}{\rho+1}}}{\rho^{\frac{\rho}{\rho+1}} \theta^{\frac{\rho}{\rho+1}}} \\
& =\left(\theta \mu^{\rho}\right)^{\frac{1}{\rho+1}}\left(\rho^{\frac{1}{\rho+1}}+\frac{1}{\rho^{\frac{\rho}{\rho+1}}}\right)=\left(\theta \mu^{\rho}\right)^{\frac{1}{\rho+1}}\left(\frac{\rho+1}{\rho^{\frac{\rho}{\rho+1}}}\right) \\
& =\left(\theta \mu^{\rho}\right)^{\frac{1}{\rho+1}}(\rho+1) \rho^{-\frac{\rho}{\rho+1}} .
\end{aligned}
$$

That is,

$$
y\left(h_{0}\right)=\left(\theta \mu^{\rho}\right)^{\frac{1}{\rho+1}}(\rho+1) \rho^{-\frac{\rho}{\rho+1}} .
$$

Consequently,

$$
y\left(h_{0}\right)=\left(\frac{K}{\Gamma(\alpha+1)}\|\| f\|\|_{\infty, \mathbb{R}}^{\alpha-1}\right)^{\frac{1}{\alpha}} \alpha(\alpha-1)^{-\left(\frac{\alpha-1}{\alpha}\right)} .
$$

We have proved that

$$
\|\|\left(f \circ g^{-1}\right)^{\prime} \circ g\|\|_{\infty, \mathbb{R}} \leq\left(\frac{K}{\Gamma(\alpha+1)}\|\| f\|\|_{\infty, \mathbb{R}}^{\alpha-1}\right)^{\frac{1}{\alpha}} \alpha(\alpha-1)^{-\left(\frac{\alpha-1}{\alpha}\right)} .
$$

Next call

$$
\xi:=4\|\| f\|\|_{\infty, \mathbb{R}}, \quad \psi=\frac{2 K}{\Gamma(\alpha+1)},
$$

both are greater than zero. Set also $\varphi:=\alpha-2>0$. We consider the function

$$
\gamma(h):=\frac{\xi}{h^{2}}+\psi h^{\varphi}=\xi h^{-2}+\psi h^{\varphi}, \forall h>0 .
$$

We have

$$
\gamma^{\prime}(h)=-2 \xi h^{-3}+\varphi \psi h^{\varphi-1}=0
$$


then

$$
\varphi \psi h^{\varphi+2}=2 \xi
$$

with a unique solution

$$
h_{0}:=h_{\text {crit. } n o}=\left(\frac{2 \xi}{\varphi \psi}\right)^{\frac{1}{\varphi+2}} .
$$

We have that

$$
\gamma^{\prime \prime}(h)=6 \xi h^{-4}+\varphi(\varphi-1) \psi h^{\varphi-2} .
$$

We see that

$$
\begin{aligned}
\gamma^{\prime \prime}\left(h_{0}\right) & =6 \xi\left(\frac{2 \xi}{\varphi \psi}\right)^{-\frac{4}{\varphi+2}}+\varphi(\varphi-1) \psi\left(\frac{2 \xi}{\varphi \psi}\right)^{\frac{(\varphi+2)-4}{\varphi+2}} \\
& =\left(\frac{2 \xi}{\varphi \psi}\right)^{-\frac{4}{\varphi+2}}[6 \xi+(\varphi-1) 2 \xi]=2 \xi\left(\frac{2 \xi}{\varphi \psi}\right)^{-\frac{4}{\varphi+2}}(\varphi+2)>0 .
\end{aligned}
$$

Therefore, $\gamma$ has a global minimum at $h_{0}=\left(\frac{2 \xi}{\varphi \psi}\right)^{\frac{1}{\varphi+2}}$, which is

$$
\begin{aligned}
\gamma\left(h_{0}\right) & =\xi\left(\frac{2 \xi}{\varphi \psi}\right)^{-\frac{2}{\varphi+2}}+\psi\left(\frac{2 \xi}{\varphi \psi}\right)^{\frac{\varphi+2-2}{\varphi+2}} \\
& =\left(\frac{2 \xi}{\varphi \psi}\right)^{-\frac{2}{\varphi+2}}\left[\xi+\psi \frac{2 \xi}{\varphi \psi}\right]=\frac{\xi}{\varphi}\left(\frac{\xi}{\varphi}\right)^{-\frac{2}{\varphi+2}}\left(\frac{2}{\psi}\right)^{-\frac{2}{\varphi+2}}(\varphi+2) \\
& =\left(\frac{\xi}{\varphi}\right)^{\frac{\varphi}{\varphi+2}}\left(\frac{\psi}{2}\right)^{\frac{2}{\varphi+2}}(\varphi+2)
\end{aligned}
$$

That is,

$$
\gamma\left(h_{0}\right)=\left(\frac{\xi}{\varphi}\right)^{\frac{\varphi}{\varphi+2}}\left(\frac{\psi}{2}\right)^{\frac{2}{\varphi+2}}(\varphi+2)
$$

Consequently,

$$
\gamma\left(h_{0}\right)=\left(\frac{4\|\| f\|\|_{\infty, \mathbb{R}}}{\alpha-2}\right)^{\frac{\alpha-2}{\alpha}}\left(\frac{K}{\Gamma(\alpha+1)}\right)^{\frac{2}{\alpha}} \alpha .
$$

We have proved that

$$
\|\|\left(f \circ g^{-1}\right)^{\prime \prime} \circ g\|\|_{\infty, \mathbb{R}} \leq\left(\frac{4\|\| f\|\|_{\infty, \mathbb{R}}}{\alpha-2}\right)^{\frac{\alpha-2}{\alpha}}\left(\frac{K}{\Gamma(\alpha+1)}\right)^{\frac{2}{\alpha}} \alpha .
$$

The theorem is established.

We also give an $L_{p}$ analog of a generalized fractional Landau inequality

Theorem 2.4. Let $p, q>1: \frac{1}{p}+\frac{1}{q}=1,2<\alpha \leq 3$ and $f \in C^{3}(\mathbb{R}, X)$, where $(X,\|\cdot\|)$ is a Banach space. Let $g \in C^{1}(\mathbb{R})$, strictly increasing, such that $g^{-1} \in C^{3}(g(\mathbb{R}))$. We assume that 
\|\|$f\|\|_{\infty, \mathbb{R}}<\infty$, and that

(56)

$$
\begin{aligned}
M:= & \max \left\{\sup _{a \in \mathbb{R}}\|\|\left(\left(D_{a+; g}^{\alpha} f\right) \circ g^{-1}\right)(z)\|\|_{p, g(\mathbb{R})},\right. \\
& \left.\sup _{a \in \mathbb{R}}\|\|\left(\left(D_{a-; g}^{\alpha} f\right) \circ g^{-1}\right)(z)\|\|_{p, g(\mathbb{R})}\right\}<\infty .
\end{aligned}
$$

Then,

1)

$$
\begin{aligned}
& \|\|\left(f \circ g^{-1}\right)^{\prime} \circ g\|\|_{\infty, \mathbb{R}} \\
& \leq\left(\alpha-\frac{1}{p}\right)\left(\frac{M}{\Gamma(\alpha)(q(\alpha-1)+1)^{\frac{1}{q}}}\right)^{\left(\frac{1}{\alpha-\frac{1}{p}}\right)}\left(\frac{\|\| f\|\|_{\infty, \mathbb{R}}}{\alpha-1-\frac{1}{p}}\right)^{\left(\frac{\alpha-1-\frac{1}{p}}{\alpha-\frac{1}{p}}\right)}
\end{aligned}
$$

2) under the additional assumption $2+\frac{1}{p}<\alpha \leq 3$, we have

(58)

$$
\begin{aligned}
& \|\|\left(f \circ g^{-1}\right)^{\prime \prime} \circ g\|\|_{\infty, \mathbb{R}} \\
& \leq\left(\alpha-\frac{1}{p}\right)\left(\frac{M}{\Gamma(\alpha)(q(\alpha-1)+1)^{\frac{1}{q}}}\right)^{\left(\frac{2}{\alpha-\frac{1}{p}}\right)}\left(\frac{4\|\| f\|\|_{\infty, \mathbb{R}}}{\alpha-2-\frac{1}{p}}\right)^{\left(\frac{\alpha-2-\frac{1}{p}}{\alpha-\frac{1}{p}}\right)} .
\end{aligned}
$$

That is,

$$
\|\|\left(f \circ g^{-1}\right)^{\prime} \circ g\|\|_{\infty, \mathbb{R}},\|\|\left(f \circ g^{-1}\right)^{\prime \prime} \circ g\|\|_{\infty, \mathbb{R}}<\infty .
$$

Proof. We continue with the proof of Theorem 2.3. By (23), we have

$$
\begin{aligned}
\|A\| & =\left\|\left(f\left(x_{1}\right)-f(a)\right)-\frac{1}{\Gamma(\alpha)} \int_{g(a)}^{g\left(x_{1}\right)}\left(g\left(x_{1}\right)-z\right)^{\alpha-1}\left(\left(D_{a+; g}^{\alpha} f\right) \circ g^{-1}\right)(z) d z\right\| \\
& \leq 2\|\| f\|\|_{\infty, \mathbb{R}}+\frac{1}{\Gamma(\alpha)} \int_{g(a)}^{g\left(x_{1}\right)}\left(g\left(x_{1}\right)-z\right)^{\alpha-1}\left\|\left(\left(D_{a+; g}^{\alpha} f\right) \circ g^{-1}\right)(z)\right\| d z \\
& \leq 2\|\| f\|\|_{\infty, \mathbb{R}}+\frac{1}{\Gamma(\alpha)}\left(\int_{g(a)}^{g\left(x_{1}\right)}\left(g\left(x_{1}\right)-z\right)^{q(\alpha-1)} d z\right)^{\frac{1}{q}} \\
& \times\left(\int_{g(a)}^{g\left(x_{1}\right)}\left\|\left(\left(D_{a+; g}^{\alpha} f\right) \circ g^{-1}\right)(z)\right\|^{p} d z\right)^{\frac{1}{p}} \\
& \leq 2\|\| f\|\|_{\infty, \mathbb{R}}+\frac{1}{\Gamma(\alpha)} \frac{\left(g\left(x_{1}\right)-g(a)\right)^{\frac{(q(\alpha-1)+1)}{q}}}{(q(\alpha-1)+1)^{\frac{1}{q}}}\|\|\left(\left(D_{a+; g}^{\alpha} f\right) \circ g^{-1}\right)(z)\|\|_{p, g(\mathbb{R})} \\
& \leq 2\|\| f\|\|_{\infty, \mathbb{R}}+\frac{1}{\Gamma(\alpha)} \frac{h^{\alpha-\frac{1}{p}}}{(q(\alpha-1)+1)^{\frac{1}{q}}}\left(\sup _{a \in \mathbb{R}}\|\|\left(\left(D_{a+; g}^{\alpha} f\right) \circ g^{-1}\right)(z)\|\|_{p, g(\mathbb{R})}\right) \\
& \leq 2\|\| f\|\|_{\infty, \mathbb{R}}+\frac{h^{\alpha-\frac{1}{p}}}{\Gamma(\alpha)}(q(\alpha-1)+1)^{\frac{1}{q}} M .
\end{aligned}
$$


That is,

(60)

$$
\|A\| \leq 2\|\| f\|\|_{\infty, \mathbb{R}}+\frac{M}{\Gamma(\alpha)(q(\alpha-1)+1)^{\frac{1}{q}}} h^{\alpha-\frac{1}{p}}, \quad h>0 .
$$

Similarly, from (24), we get

$$
\begin{aligned}
\|B\| & =\left\|\left(f\left(x_{2}\right)-f(a)\right)-\frac{1}{\Gamma(\alpha)} \int_{g\left(x_{2}\right)}^{g(a)}\left(z-g\left(x_{2}\right)\right)^{\alpha-1}\left(\left(D_{a-; g}^{\alpha} f\right) \circ g^{-1}\right)(z) d z\right\| \\
& \leq 2\|\| f\|\|_{\infty, \mathbb{R}}+\frac{1}{\Gamma(\alpha)} \int_{g\left(x_{2}\right)}^{g(a)}\left(z-g\left(x_{2}\right)\right)^{\alpha-1}\left\|\left(\left(D_{a-; g}^{\alpha} f\right) \circ g^{-1}\right)(z)\right\| d z \\
& \leq 2\|\| f\|\|_{\infty, \mathbb{R}}+\frac{1}{\Gamma(\alpha)}\left(\int_{g\left(x_{2}\right)}^{g(a)}\left(z-g\left(x_{2}\right)\right)^{q(\alpha-1)} d z\right)^{\frac{1}{q}} \\
& \times\left(\int_{g\left(x_{2}\right)}^{g(a)}\left\|\left(\left(D_{a-; g}^{\alpha} f\right) \circ g^{-1}\right)(z)\right\|^{p} d z\right)^{\frac{1}{p}} \\
& \leq 2\|\| f\|\|_{\infty, \mathbb{R}}+\frac{1}{\Gamma(\alpha)} \frac{\left(g(a)-g\left(x_{2}\right)\right)^{\alpha-\frac{1}{p}}}{(q(\alpha-1)+1)^{\frac{1}{q}}}\left(\sup _{a \in \mathbb{R}}\|\|\left(\left(D_{a-; g}^{\alpha} f\right) \circ g^{-1}\right)(z)\|\|_{p, g(\mathbb{R})}\right) \\
& \leq 2\|\| f\|\|_{\infty, \mathbb{R}}+\frac{h^{\alpha-\frac{1}{p}}}{\Gamma(\alpha)}(q(\alpha-1)+1)^{\frac{1}{q}} M .
\end{aligned}
$$

(61)

That is,

(62)

$$
\|B\| \leq 2\|\| f\|\|_{\infty, \mathbb{R}}+\frac{M}{\Gamma(\alpha)(q(\alpha-1)+1)^{\frac{1}{q}}} h^{\alpha-\frac{1}{p}}, \quad h>0 .
$$

Hence, it holds

$$
\begin{aligned}
\|A+B\| & \leq\|A\|+\|B\| \\
& \stackrel{(\text { by (60), (62)) }}{\leq} 4\|\| f\|\|_{\infty, \mathbb{R}}+\frac{2 M}{\Gamma(\alpha)(q(\alpha-1)+1)^{\frac{1}{q}}} h^{\alpha-\frac{1}{p}}, \quad h>0 .
\end{aligned}
$$

Furthermore, we have

$$
\begin{aligned}
\|A-B\| & \stackrel{(34)}{\leq} 2\|\| f\|\|_{\infty, \mathbb{R}} \\
& +\frac{1}{\Gamma(\alpha)}\left[\left(\int_{g(a)}^{g\left(x_{1}\right)}\left(g\left(x_{1}\right)-z\right)^{q(\alpha-1)} d z\right)^{\frac{1}{q}}\|\|\left(\left(D_{a+; g}^{\alpha} f\right) \circ g^{-1}\right)(z)\|\|_{p, g(\mathbb{R})}\right. \\
& \left.+\left(\int_{g\left(x_{2}\right)}^{g(a)}\left(z-g\left(x_{2}\right)\right)^{q(\alpha-1)} d z\right)^{\frac{1}{q}}\|\|\left(\left(D_{a-; g}^{\alpha} f\right) \circ g^{-1}\right)(z)\|\|_{p, g(\mathbb{R})}\right] \\
& \leq 2\|\| f\|\|_{\infty, \mathbb{R}}+\frac{M}{\Gamma(\alpha)}\left[\frac{2 h^{\alpha-\frac{1}{p}}}{(q(\alpha-1)+1)^{\frac{1}{q}}}\right]
\end{aligned}
$$

We have proved that

$$
\frac{\|A-B\|}{2} \leq\|\| f\|\|_{\infty, \mathbb{R}}+\frac{M}{\Gamma(\alpha)(q(\alpha-1)+1)^{\frac{1}{q}}} h^{\alpha-\frac{1}{p}}, \quad h>0 .
$$


From (27), (65), we have

(66)

$$
\left\|\left(f \circ g^{-1}\right)^{\prime}(g(a))\right\| \leq \frac{\|\| f\|\|_{\infty, \mathbb{R}}}{h}+\frac{M}{\Gamma(\alpha)(q(\alpha-1)+1)^{\frac{1}{q}}} h^{\alpha-\frac{1}{p}-1},
$$

$h>0$, any $a \in \mathbb{R}$. And from (27), (63), we get that

$$
\left\|\left(f \circ g^{-1}\right)^{\prime \prime}(g(a))\right\| \leq \frac{4\|\| f\|\|_{\infty, \mathbb{R}}}{h^{2}}+\frac{2 M}{\Gamma(\alpha)(q(\alpha-1)+1)^{\frac{1}{q}}} h^{\alpha-\frac{1}{p}-2},
$$

$h>0$, any $a \in \mathbb{R}$. Hence,

$$
\|\|\left(f \circ g^{-1}\right)^{\prime} \circ g\|\|_{\infty, \mathbb{R}} \leq \frac{\|\| f\|\|_{\infty, \mathbb{R}}}{h}+\left(\frac{M}{\Gamma(\alpha)(q(\alpha-1)+1)^{\frac{1}{q}}}\right) h^{\left(\alpha-\frac{1}{p}-1\right)}
$$

and

(69)

$$
\|\|\left(f \circ g^{-1}\right)^{\prime \prime} \circ g\|\|_{\infty, \mathbb{R}} \leq \frac{4\|\| f\|\|_{\infty, \mathbb{R}}}{h^{2}}+\left(\frac{2 M}{\Gamma(\alpha)(q(\alpha-1)+1)^{\frac{1}{q}}}\right) h^{\left(\alpha-\frac{1}{p}-2\right)},
$$

true $\forall h>0,2<\alpha \leq 3, p, q>1: \frac{1}{p}+\frac{1}{q}=1$. In (69), we restrict ourselves to $2+\frac{1}{p}<\alpha \leq 3$. Call

$$
\mu:=\|\| f\|\|_{\infty, \mathbb{R}}, \quad \theta=\frac{M}{\Gamma(\alpha)(q(\alpha-1)+1)^{\frac{1}{q}}},
$$

both are greater than zero. Set also $\rho:=\alpha-1-\frac{1}{p}>\frac{1}{q}>0$. We consider the function

$$
y(h):=\frac{\mu}{h}+\theta h^{\rho}, \quad \forall h>0 .
$$

As in the proof of Theorem 2.3, it has only one critical number

$$
h_{0}:=h_{\text {crit.no }}=\left(\frac{\mu}{\rho \theta}\right)^{\frac{1}{\rho+1}}
$$

and a global minimum

$$
y\left(h_{0}\right)=\theta^{\frac{1}{\rho+1}} \mu^{\frac{\rho}{\rho+1}}(\rho+1) \rho^{-\frac{\rho}{\rho+1}} .
$$

Consequently,

(74)

$$
\begin{aligned}
y\left(h_{0}\right)= & \left(\frac{M}{\Gamma(\alpha)(q(\alpha-1)+1)^{\frac{1}{q}}}\right)^{\frac{1}{\left(\alpha-\frac{1}{p}\right)}}\left(\|\| f \|_{\infty, \mathbb{R}}\right)^{\left(\frac{\alpha-1-\frac{1}{p}}{\alpha-\frac{1}{p}}\right)} \\
& \left(\alpha-\frac{1}{p}\right)\left(\alpha-1-\frac{1}{p}\right)^{-\left(\frac{\alpha-1-\frac{1}{p}}{\alpha-\frac{1}{p}}\right)} .
\end{aligned}
$$

We have proved that (see (68))

$$
\begin{aligned}
& \|\|\left(f \circ g^{-1}\right)^{\prime} \circ g\|\|_{\infty, \mathbb{R}} \\
\leq & \left(\frac{M}{\Gamma(\alpha)(q(\alpha-1)+1)^{\frac{1}{q}}}\right)^{\frac{1}{\left(\alpha-\frac{1}{p}\right)}}\left(\frac{\|\| f\|\|_{\infty, \mathbb{R}}}{\alpha-1-\frac{1}{p}}\right)^{\left(\frac{\alpha-1-\frac{1}{p}}{\alpha-\frac{1}{p}}\right)}\left(\alpha-\frac{1}{p}\right) .
\end{aligned}
$$

We also call

$$
\xi:=4\|\| f\|\|_{\infty, \mathbb{R}}, \quad \psi=\frac{2 M}{\Gamma(\alpha)(q(\alpha-1)+1)^{\frac{1}{q}}},
$$


both are greater than zero. Set also $\varphi:=\alpha-2-\frac{1}{p}>0$. We consider the function

$$
\gamma(h):=\frac{\xi}{h^{2}}+\psi h^{\varphi}, \forall h>0 .
$$

As in the proof of Theorem 2.3, $\gamma$ has a global minimum at

$$
h_{0}=\left(\frac{2 \xi}{\varphi \psi}\right)^{\frac{1}{\varphi+2}}
$$

which is

$$
\gamma\left(h_{0}\right)=\left(\frac{\xi}{\varphi}\right)^{\frac{\varphi}{\varphi+2}}\left(\frac{\psi}{2}\right)^{\frac{2}{\varphi+2}}(\varphi+2)
$$

Consequently,

$$
\gamma\left(h_{0}\right)=\left(\frac{4\|\| f\|\|_{\infty, \mathbb{R}}}{\alpha-2-\frac{1}{p}}\right)^{\left(\frac{\alpha-2-\frac{1}{p}}{\alpha-\frac{1}{p}}\right)}\left(\frac{M}{\Gamma(\alpha)(q(\alpha-1)+1)^{\frac{1}{q}}}\right)^{\left(\frac{2}{\alpha-\frac{1}{p}}\right)}\left(\alpha-\frac{1}{p}\right) .
$$

We have proved that (see (69))

$$
\begin{aligned}
& \|\|\left(f \circ g^{-1}\right)^{\prime \prime} \circ g\|\|_{\infty, \mathbb{R}} \\
\leq & \left(\frac{4\|\| f\|\|_{\infty, \mathbb{R}}}{\alpha-2-\frac{1}{p}}\right)^{\left(\frac{\alpha-2-\frac{1}{p}}{\alpha-\frac{1}{p}}\right)}\left(\frac{M}{\Gamma(\alpha)(q(\alpha-1)+1)^{\frac{1}{q}}}\right)^{\left(\frac{2}{\alpha-\frac{1}{p}}\right)}\left(\alpha-\frac{1}{p}\right) .
\end{aligned}
$$

The theorem is established.

Next, we apply Theorems 2.3, 2.4 for $g(t)=e^{t}, t \in \mathbb{R}$ and $\alpha=2.5$.

Corollary 2.1. Let $f \in C^{3}(\mathbb{R}, X)$, where $(X,\|\cdot\|)$ is a Banach space. We assume that \|\|$f\|\|_{\infty, \mathbb{R}}<\infty$, and that

$$
\begin{aligned}
K_{2.5}:= & \max \left\{\|\|\left(\left(D_{a+; e^{t}}^{2.5} f\right) \circ \ln \right)(z)\|\|_{\infty, \mathbb{R} \times(0, \infty)},\right. \\
& \left.\|\|\left(\left(D_{a-; e^{t}}^{2.5} f\right) \circ \ln \right)(z)\|\|_{\infty, \mathbb{R} \times(0, \infty)}\right\}<\infty,
\end{aligned}
$$

where $(a, z) \in \mathbb{R} \times(0, \infty)$. Then,

$$
\|\|(f \circ \ln )^{\prime} \circ e^{t}\|\|_{\infty, \mathbb{R}} \leq 1.21136\left(K_{2.5}\right)^{0.4}\left(\|\| f\|\|_{\infty, \mathbb{R}}\right)^{0.6}
$$

and

$$
\|\|(f \circ \ln )^{\prime \prime} \circ e^{t}\|\|_{\infty, \mathbb{R}} \leq 1.44713\left(K_{2.5}\right)^{0.8}\left(\|\| f\|\|_{\infty, \mathbb{R}}\right)^{0.2} .
$$

That is,

$$
\|\|(f \circ \ln )^{\prime} \circ e^{t}\|\|_{\infty, \mathbb{R}},\|\|(f \circ \ln )^{\prime \prime} \circ e^{t}\|\|_{\infty, \mathbb{R}}<\infty .
$$

Proof. By Theorem 2.3.

We finish with the following result. 
Corollary 2.2. (case of $g(t)=e^{t}, \alpha=2.5, p=q=2$ ) Let $f \in C^{3}(\mathbb{R}, X)$, where $(X,\|\cdot\|)$ is a Banach space. We assume that \|\|$f\|\|_{\infty, \mathbb{R}}<\infty$, and that

$$
\begin{aligned}
M_{2.5}:= & \max \left\{\sup _{a \in \mathbb{R}}\|\|\left(\left(D_{a+; e^{t}}^{2.5} f\right) \circ \ln \right)(z)\|\|_{2,(0, \infty)},\right. \\
& \left.\sup _{a \in \mathbb{R}}\|\|\left(\left(D_{a-; e^{t}}^{2.5} f\right) \circ \ln \right)(z)\|\|_{2,(0, \infty)}\right\}<\infty .
\end{aligned}
$$

Then,

$$
\|\|(f \circ \ln )^{\prime} \circ e^{t}\|\|_{\infty, \mathbb{R}} \leq 1.226583057 \sqrt{M_{2.5}\|\| f\|\|_{\infty, \mathbb{R}}}<\infty .
$$

Proof. By Theorem 2.4, (57).

\section{REFERENCES}

[1] A. Aglic Aljinovic, Lj. Marangunic and J. Pecaric: On Landau type inequalities via Ostrowski inequalities, Nonlinear Funct. Anal. Appl. Vol., 10, 4 (2005), pp. 565-579.

[2] G. Anastassiou: Fractional Differentiation inequalities, Research monograph, Springer, New York, 2009.

[3] G. A. Anastassiou: Advances on Fractional Inequalities, Springer, New York, 2011.

[4] G. A. Anastassiou: Intelligent Computations: Abstract Fractional Calculus, Inequalities, Approximations, Springer, Heidelberg, New York, 2018.

[5] N. S. Barnett, S. S. Dragomir: Some Landau type inequalities for functions whose derivatives are of locally bounded variation, Tamkang Journal of Mathematics, 37, No. 4, 301-308, winter 2006.

[6] Z. Ditzian: Remarks, questions and conjectures on Landau-Kolmogorov-type inequalities, Math. Inequal. Appl., 3 (2000), $15-24$.

[7] G. H. Hardy, J. E. Littlewood: Some integral inequalities connected with the calculus of variations, Quart. J. Math. Oxford Ser., 3 (1932), 241-252.

[8] G. H. Hardy, E. Landau and J. E. Littlewood: Some inequalities satisfied by the integrals or derivatives of real or analytic functions, Math. Z., 39 (1935), 677-695.

[9] R. R. Kallman, G. C. Rota: On the inequality $\left\|f^{\prime}\right\|^{2} \leq 4\|f\| \cdot\left\|f^{\prime \prime}\right\|$, in Inequalities, Vol. II, (O. Shisha, Ed.), 187-192, Academic Press, New York, 1970.

[10] E. Landau: Einige Ungleichungen für zweimal differentzierban funktionen, Proc. London Math. Soc., 13 (1913), 43-49.

[11] J. Mikusinski: The Bochner integral, Academic Press, New York, 1978.

[12] G. E. Shilov: Elementary Functional Analysis, Dover Publications Inc., New York, 1996.

George A. AnAstassiou

UNIVERSITY OF MEMPHIS

Department of Mathematical SCiences

MEMPHIS, TN 38152, U.S.A.

ORCID: 0000-0002-3781-9824

E-mail address: ganastss@memphis.edu 